\title{
Collecting Hand Wipe Samples to Assess Thirdhand Smoke Exposure
}

\author{
E. Melinda Mahabee-Gittens ${ }^{1 *}$, Penelope J. E. Quintana ${ }^{2}$, Eunha Hoh ${ }^{2}$, \\ Ashley L. Merianos ${ }^{3}$, Lara Stone ${ }^{1}$, Nicolas Lopez-Galvez ${ }^{2}$ and Georg E. Matt ${ }^{4}$ \\ ${ }^{1}$ Division of Emergency Medicine, Cincinnati Children's Hospital Medical Center, Department of Pediatrics, University of \\ Cincinnati College of Medicine, Cincinnati, OH, United States, ${ }^{2}$ School of Public Health, San Diego State University, San \\ Diego, CA, United States, ${ }^{3}$ School of Human Services, University of Cincinnati, Cincinnati, OH, United States, ${ }^{4}$ Department \\ of Psychology, San Diego State University, San Diego, CA, United States
}

Keywords: nicotine, secondhand smoke exposure, thirdhand smoke exposure, thirdhand smoke pollution children, hand wipes, dermal exposure

\section{INTRODUCTION}

Thirdhand smoke (THS) is the persistent toxic residue of tobacco smoke pollutants that attaches to surfaces, remains in dust, and becomes embedded in environments long after secondhand smoke (SHS) has been released into the air (1). THS pollutants include harmful toxicants such as nicotine, tobacco-specific nitrosamines, polycyclic aromatic hydrocarbons, heavy metals, and other semi-volatile compounds (1). Unlike children's and adult's episodic and variable exposure to SHS, exposure to THS is chronic and may be cumulative over days to years (1-3). Compared

OPEN ACCESS

Edited by:

Dimirios Nikolopoulos,

University of West Attica, Greece

Reviewed by:

Hanna Danielewicz,

Wroclaw Medical University, Poland

Myung-Bae Park,

Pai Chai University, South Korea

*Correspondence:

E. Melinda Mahabee-Gittens melinda.mahabee-gittens@cchmc.org

Specialty section:

This article was submitted to Environmental health and Exposome, a section of the journal Frontiers in Public Health

Received: 03 September 2021 Accepted: 02 December 2021 Published: 20 December 2021

Citation:

Mahabee-Gittens EM, Quintana PJE, Hoh E, Merianos AL, Stone L, Lopez-Galvez N and Matt GE (2021) Collecting Hand Wipe Samples to Assess Thirdhand Smoke Exposure. Front. Public Health 9:770505. do: 10.3389/fpubh.2021.770505 to adults, children are more susceptible to inhaling, ingesting, and dermally absorbing THS due to their smaller size, hand-to-mouth behaviors, developing organs, and other varying biological characteristics (4). THS residue from previously smoked tobacco in children's homes or cars creates a pervasive reservoir of THS pollutants on carpets, furniture, clothes, and toys, and in dust $(1,2)$. Thus, non-smoking children's and adults' overall tobacco smoke exposure (TSE) consists of SHS and THS exposure if they live with tobacco product users or THS exposure only if they do not live with tobacco product users but live in THS-polluted environments (e.g., multiunit homes) $(5,6)$. Cotinine is a metabolite of nicotine $(7)$, and it can be measured in urine, saliva, blood, hair, and nails (8). Cotinine is the biomarker most frequently measured to assess non-smokers' overall TSE from SHS and THS. However, since cotinine is not specific to THS, cotinine levels cannot be used to determine if non-smokers' TSE includes exposure to SHS and THS or THS alone. Further, cotinine levels are affected by factors other than non-smokers' nicotine exposure such as their age, sex, and genetic factors $(7,9)$. Although there is no gold standard marker of THS exposure, since THS pollution in non-smokers' environments can be dermally absorbed, nicotine levels on hands serve as a proxy of non-smokers' THS exposure (10-13).

We believe that hand nicotine levels are preferable when measuring non-smokers' exposure to tobacco smoke pollutants from THS compared to cotinine (12). Our team was the first to collect and analyze hand wipes from children in the hospital setting (11). Our results indicated that smokers' children bring nicotine, a THS pollutant, on their hands into the hospital setting where no one is smoking. Hand nicotine levels are highly correlated with children's cotinine levels (11-13), and levels may be associated with clinical illnesses $(10,12)$, but further research is warranted. Clinical researchers who are studying the health effects of child or adult TSE and ways to reduce or eliminate TSE should consider obtaining hand wipes as a way to assess children's or adults' THS exposure. The inclusion of hand nicotine levels in TSE research will help determine: (1) the prevalence of THS exposure in non-smokers; (2) the associations between THS exposure and clinical illnesses; (3) sources of THS pollution that should be targeted in remediation efforts; and (4) sociodemographic differences in THS exposure patterns and potentially modifiable risk factors (e.g., lack of smoking bans) that can be targeted in TSE interventions (see Figure 1). 


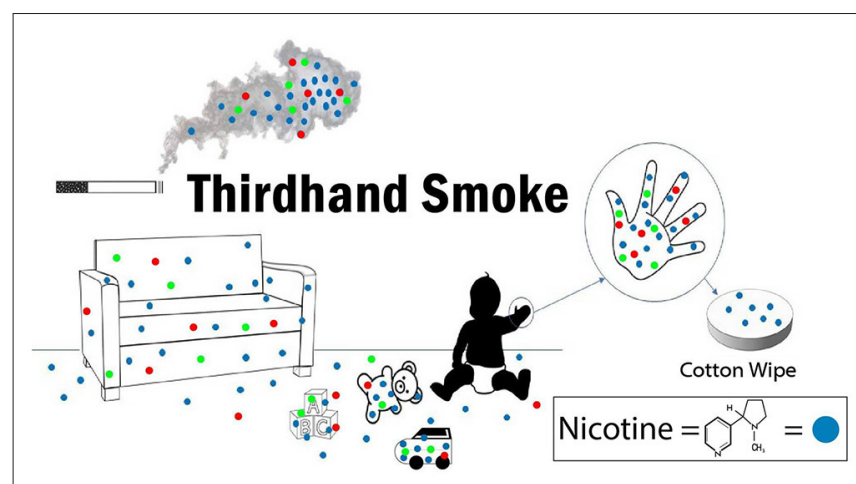

FIGURE 1 | Graphical depiction of thirdhand smoke and hand wipe collection.

In this paper, we present methods that represent best practices to collect hand wipes from child or adult research participants in clinical and non-clinical settings. Recommended quality control/quality assurance practices that should be used are also presented to ensure the samples are collected correctly and that the measurements obtained represent reliable and valid nicotine levels.

\section{MATERIALS AND METHODS}

\section{Preparation for Hand Wipe Collection Pre-screening Wipes}

Wipes must be prescreened by the clinical research coordinator (CRC) before use. Purchase 100\% cotton facial wipes (dimensions $9.5 \times 6.7 \times 3.9$ inches; 2.56 ounces; e.g., 80 pack of Swisspers Cotton Organic Rounds). For each purchase, select $10 \%$ from the pack of wipes and measure for nicotine with isotope-dilution liquid chromatography with tandem mass spectrometry (LCMS/MS) (14) with a limit of detection (LOD) at 0.19 nanogram (ng)/wipe (12). Acceptable levels of contamination in each wipe screened from the batch are $<1 \mathrm{ng} /$ wipe. If contamination levels in the screened sample wipes are higher than that, discard the batch and repurchase wipes.

\section{Baking Amber Sample Collection Vials}

Wash vials with water and detergent. Then, cover the mouths of the vials with aluminum foil, place in a laboratory oven, and bake at $450^{\circ} \mathrm{C}$ for $6 \mathrm{~h}$. Rinse Teflon caps with methanol three times, let dry, and close the vials with cleaned Teflon caps affixed to them.

\section{Hand Wipe Sampling Kit Assembly}

Sampling kits are prepared prior to use in a clean area while wearing gloves. For each hand wipe sample planned, the kits should contain:

1. Gloves (acrylonitrile), $\geq 2$ pairs

2. One piece of $12 " \times 18$ " aluminum foil

3. Two $100 \%$ pre-screened cotton facial wipes (see section Prescreening Wipes above)

4. Two polystyrene petri dishes [diameter: $100 \mathrm{~mm}$, height: 15 mm; FisherScientific Catolog (FisherSci Cat) \#: FB0875712]
5. Two 2 milliliter $(\mathrm{ml})$ sterile plastic disposable transfer pipettes (FisherSci Cat \#: 137119D)

6. Two $20 \mathrm{ml}$ Environmental Protection Agency Screw amber glass vials with Teflon-lined caps (FisherSci Cat \#: 03-377-36)

7. One $15 \mathrm{ml}$ sterile polypropylene centrifuge tube with $10 \mathrm{ml}$ distilled water (FisherSci cat\#: 07-201-330)

8. One $15 \mathrm{ml}$ sterile polypropylene centrifuge tube with 0.10 grams ascorbic acid (FisherSci Cat \# 50-213-151), weighed out on a microbalance

9. Tube rack

10. One-gallon self-sealing plastic bag.

\section{Hand Wipe Sample Collection Preparation of Wipes}

Follow these procedures up to $48 \mathrm{~h}$ prior to hand wipe sample collection. At the end of this preparation, CRCs will have a petri dish with two wipes ready to deploy and sample collection vials readied for use if these steps are followed:

1. In a clean work area out of reach of children, set up the sample material workstation by placing aluminum foil on this clean surface.

2. Put on a new pair of gloves and put the tube of distilled water and the tube with ascorbic acid side-by-side in the rack and loosen the caps. Remove a transfer pipette from the wrapper and hold it in the dominant hand and with the non-dominant hand, remove and hold the cap from the water tube. Draw $1 \mathrm{ml}$ of distilled water into the pipette. Open the ascorbic acid tube, hold the cap in the non-dominant hand, and squeeze distilled water into the tube. Repeat these steps until there are $10 \mathrm{ml}$ of distilled water in the tube with the ascorbic acid. Replace and tighten the cap and invert the tube repeatedly to completely dissolve the powder.

3. Unwrap another transfer pipette, open a petri dish with the two cotton rounds, and place the lid on the table, open side up. Using the pipette, squirt $1 \mathrm{ml}$ of ascorbic acid solution onto one cotton round; repeat with an additional $1 \mathrm{ml}$ of solution. Repeat steps to squirt each cotton round with a total of $2 \mathrm{ml}$ ascorbic acid solution; wipes are stable for at least $8 \mathrm{~h}$.

4. Remove two amber vials from the plastic bag, place on the table, remove the aluminum foil from the amber vial, discard the foil, and retighten the lids. Using a permanent marker and pencil, label one of the vials as "blank" and the other as "hand wipe" with a number and date that matches the sample number of the hand wipe sample.

\section{Obtaining the Hand Wipe Samples}

Before entering the participant's room, the CRC will need the prepared wipes and sample vials, gloves, a data sheet to record sample collection, signed informed consent documents as needed, and a questionnaire.

\section{Blank Collection}

Put on a new pair of gloves, set the amber vial labeled as "blank" on the table, remove the Teflon cap, and set the cap face up on the table. Open the petri dish, place the cotton round into the amber vial, replace the cap, and place the vial in the plastic bag. 


\section{Hand Wipe Sample Collection}

Set the amber vial labeled "hand wipe" on the table, remove the Teflon cap, and set the cap face up on the table. Grasp the wipe firmly in the gloved hand and prepare to obtain the hand wipe sample from the participant's dominant hand, or, if the participant is too young to determine the dominant hand, the right hand is used. Sample the palm of the hand and volar aspect (inward facing part) of all fingers. Wipe from the wrist to the base of the fingers (knuckles). Use five strokes, starting from the base. Next, wipe the fingers and thumbs, including the finger web areas and sides of the fingers, followed by the fingertips. Place the cotton round in the amber vial, replace the cap, and place the vial in the plastic bag.

\section{Hand Measurement}

Obtain the participant's hand measurements using measurement digital calipers (e.g., Adoric 0-6" Calipers Measuring ToolElectronic Micrometer Caliper). Measure from the base of the palm to the tip of the middle finger (length) and from the width of the palm without including the thumb; record measurements in centimeters on the data collection sheet.

\section{Questionnaire}

In the questionnaire, ask the caregiver, child, and/or adult participant the last time that hand washing occurred (including what was used, e.g., soap and sanitizer) and/or the last time that lotion or any other products were put on the participant's hands; record the information on the data collection sheet.

\section{Labeling, Storage, and Shipment}

Record the date and time of hand wipe collection and sample numbers and submerge the plastic bag containing the samples in a cup of ice. Collected samples and field blanks should be placed on ice in a cooler and transported as quickly as possible (within $4 \mathrm{~h}$ ) to a freezer where they can be stored at $\leq-20^{\circ}$ Celsius until shipment and/or analysis. Ship samples and blanks on dry ice via overnight mail to the laboratory for analysis. Nicotine analysis of hand wipes by isotope dilution LC-MS/MS has previously been described (14).

\section{SPECIAL CONSIDERATIONS FOR BUSY PEDIATRIC CLINICAL SETTINGS}

Collecting hand wipes during clinical visits can be challenging but feasible with planning. To facilitate hand wipe collection, the CRC should have all materials and collection vials prepared and labeled before entering the participant's room. It is important to clean the surface that will be used to set up the collection materials and place the wipes as far from caregivers, children, and/or adult participants as possible. The CRC should try and make the collection procedure a fun "game" for children by asking them to help count their fingers and other tactics. Although caregivers may wish to help with the sample collection, it is important to avoid this as parents may have nicotine on their own hands. Ideally, hand wipes should be collected prior to the child washing or sanitizing their hands.
This may be difficult when frequent hand washing and the use of hand sanitizers are being employed to decrease the spread of the severe acute respiratory syndrome coronavirus2 (SARS-CoV-2), the virus which causes coronavirus disease 2019 (COVID-19) (15); thus it is important to ask parents what hand hygiene methods have been used prior to hand wipe collection.

\section{REPORTING HAND NICOTINE LEVELS}

Field blank values (ng nicotine) should be subtracted from the hand wipe sample values in ng before reporting results. Analyzing field blanks that are equal to $\geq 20 \%$ of the total sample size is acceptable. The average of the analyzed field blanks in ng nicotine should be calculated for each analysis batch and the average value subtracted from hand wipe sample results. Field blank values may be higher for children with TSE than those unexposed (16). However, if field blank levels exceed $5 \mathrm{ng} /$ wipe, investigators should review data collection practices, transportation, sample handling, and sample analysis to consider changes to reduce sample contamination. Results are reported in $\mathrm{ng}$ per hand wipe or $\mathrm{ng}$ per $\mathrm{m}^{2}$ if hand surface area is adjusted for when hand measurements are available.

\section{SAMPLE RANGES OF HAND NICOTINE AND FIELD BLANK LEVELS ON CHILDREN OF TOBACCO PRODUCT USERS IN RESEARCH}

In a previous study (12), hand wipe samples were collected from 276 pediatric emergency department patients [Median $(\mathrm{Mdn})$ age $=4.0$ years] who lived with at least one adult who smoked cigarettes. All children had detectable hand nicotine levels ranging from 4.0 to $2,191.3 \mathrm{ng} /$ wipe; Geometric Mean = 89.7 ng/wipe; 95\% Confidence Interval $=(78.9 ; 102.0), \mathrm{Mdn}=102.6 \mathrm{ng} /$ wipe, Interquartile range $=46.4-181.9$. Field blanks were analyzed to adjust for potential contamination of the wipe samples; the Mdn for the field blanks was $1.8 \mathrm{ng}$ nicotine/wipe and field bank corrections ranged from 0 to $16.7 \mathrm{ng}$ nicotine/wipe for the 276 children.

\section{SUMMARY}

This paper provides a collection method for obtaining hand wipes in children or adults that can later be analyzed for nicotine, a THS pollutant. By obtaining field blanks to adjust for potential environmental contamination with nicotine, accurate measures of hand nicotine levels can be obtained. Assuring complete contents of sample collection kits, detailed directions for sampling participants' hands, and immediate sample storage at $\leq-20^{\circ} \mathrm{C}$ will facilitate proper collection and ensure sample integrity. The methods described can be translated to pediatric and adult populations in a variety of settings including clinical settings such as the emergency department, 
urgent care departments, and hospitals, as well as non-clinical settings such as homes and workplaces. We strongly recommend obtaining hand wipe nicotine levels in clinical research studies to obtain a more complete picture of children's and adults' overall TSE.

\section{AUTHOR CONTRIBUTIONS}

EM-G and GM conceptualized the article, drafted the manuscript, and approved the final manuscript as submitted. PQ, $\mathrm{EH}, \mathrm{AM}, \mathrm{LS}$, and NL-G drafted the manuscript and approved the final manuscript as submitted. All authors contributed to the article and approved the submitted version.

\section{REFERENCES}

1. Jacob P, III, Benowitz NL, Destaillats H, Gundel L, Hang B, Martins-Green M, et al. Thirdhand smoke: new evidence, challenges, and future directions. Chem Res Toxicol. (2017) 30:270-94. (In Eng). doi: 10.1021/acs.chemrestox.6b00343

2. Matt GE, Quintana PJ, Destaillats H, Gundel LA, Sleiman M, Singer BC, et al. Thirdhand tobacco smoke: emerging evidence and arguments for a multidisciplinary research agenda. Environ Health Persp. (2011) 119:1218-26. (In English). doi: 10.1289/ehp.1103500

3. Diez-Izquierdo A, Cassanello-Penarroya P, Lidon-Moyano C, MatillaSantander N, Balaguer A, Martinez-Sanchez JM. Update on Thirdhand smoke: a comprehensive systematic review. Environ Res. (2018) 167:341-71. (In Eng). doi: 10.1016/j.envres.2018.07.020

4. World Health Organization. Summary of Principles for Evaluating Health Risks in Children Associated with Exposure to Chemicals. Geneva, Switzerland: World Health Organizaiton (2011).

5. Matt GE, Quintana PJ, Zakarian JM, Fortmann AL, Chatfield DA, Hoh E, et al. When smokers move out and non-smokers move in: residential Thirdhand smoke pollution and exposure. Tob Control. (2011) 20:e1. (In English). doi: 10.1136/tc.2010.037382

6. Matt GE, Quintana PJE, Zakarian JM, Hoh E, Hovell MF, MahabeeGittens $\mathrm{M}$, et al. When smokers quit: exposure to nicotine and carcinogens persists from Thirdhand smoke pollution. Tob Control. (2016) 26:548-556. doi: 10.1136/tobaccocontrol-2016-053119

7. Benowitz NL, Hukkanen J, Jacob P, III. Nicotine chemistry, metabolism, kinetics and biomarkers. Handb Exp Pharmacol. (2009) 192:29-60. doi: 10.1007/978-3-540-69248-5_2

8. Avila-Tang E, Al-Delaimy WK, Ashley DL, Benowitz N, Bernert JT, Kim $\mathrm{S}$, et al. Assessing secondhand smoke using biological markers. Tob Control. (2013) 22:164-71. (In Eng). doi: 10.1136/tobaccocontrol-2011-0 50298

9. Zhu AZ, Renner CC, Hatsukami DK, Swan GE, Lerman C, Benowitz NL, et al. The ability of plasma cotinine to predict nicotine and carcinogen exposure is altered by differences in CYP2A6: the influence of genetics, race, and sex. Cancer Epidemiol Biomarkers Prev. (2013) 22:708-18. doi: 10.1158/1055-9965.EPI-12-1234-T

10. Mahabee-Gittens EM, Merianos AL, Hoh E, Quintana PJ, Matt GE. Nicotine on children's hands: limited protection of smoking bans and initial clinical findings. Tob Use Insights. (2019) 12:1179173X18823493. doi: 10.1177/1179173X188 23493

\section{FUNDING}

This work was supported in part by the National Institute of Environmental Health Sciences (NIH Grant Numbers R01ES030743, R01ES027815, and R21ES032161), and the National Institute on Drug Abuse (NIH Grant Number K01DA044313).

\section{ACKNOWLEDGMENTS}

The investigators thank Mr. Nicholas Gittens for his work on the figure. The investigators also thank the study participants and study staff who have made this research possible.

11. Mahabee-Gittens EM, Merianos AL, Matt GE. Preliminary evidence that high levels of nicotine on children's hands may contribute to overall tobacco smoke exposure. Tob Control. (2018) 27:217-9. (In English). doi: 10.1136/tobaccocontrol-2016-053602

12. Mahabee-Gittens EM, Merianos AL, Jandarov RA, Quintana PJE, Hoh E, Matt GE. Differential associations of hand nicotine and urinary cotinine with children's exposure to tobacco smoke and clinical outcomes. Environ Res. (2021) 202:111722. doi: 10.1016/j.envres.2021.111722

13. Mahabee-Gittens EM, Matt GE, Jandarov RJ, Merianos AL. Hand nicotine and cotinine in children exposed to cigars: a pilot study. Tob Regul Sci. (2021) 7:170-6. (Article) (In English). doi: 10.18001/TRS.7.3.2

14. Matt GE, Quintana PJE, Hoh E, Zakarian JM, Dodder NG, Record RA, et al. Remediating Thirdhand smoke pollution in multiunit housing: temporary reductions and the challenges of persistent reservoirs. Nicotine Tob Res. (2021) 23:364-372. doi: 10.1093/ntr/ntaa151

15. Gupta S, Smith L, Diakiw A. Avoidance of COVID-19 for Children and Adolescents and Isolation Precautions. Pediatr Clin North Am. (2021) 68:1103-1118. doi: 10.1016/j.pcl.2021.05.011

16. Quintana PJ, Matt GE, Chatfield D, Zakarian JM, Fortmann AL, Hoh E. Wipe sampling for nicotine as a marker of Thirdhand tobacco smoke contamination on surfaces in homes, cars, and hotels. Nicotine Tob Res. (2013) 15:1555-63. doi: $10.1093 / \mathrm{ntr} / \mathrm{ntt} 014$

Conflict of Interest: The authors declare that the research was conducted in the absence of any commercial or financial relationships that could be construed as a potential conflict of interest.

Publisher's Note: All claims expressed in this article are solely those of the authors and do not necessarily represent those of their affiliated organizations, or those of the publisher, the editors and the reviewers. Any product that may be evaluated in this article, or claim that may be made by its manufacturer, is not guaranteed or endorsed by the publisher.

Copyright (C) 2021 Mahabee-Gittens, Quintana, Hoh, Merianos, Stone, Lopez-Galvez and Matt. This is an open-access article distributed under the terms of the Creative Commons Attribution License (CC BY). The use, distribution or reproduction in other forums is permitted, provided the original author(s) and the copyright owner(s) are credited and that the original publication in this journal is cited, in accordance with accepted academic practice. No use, distribution or reproduction is permitted which does not comply with these terms. 